EPJ Web of Conferences 28, 12013 (2012)

DOI: $10.1051 /$ epjconf/20122812013

(C) Owned by the authors, published by EDP Sciences, 2012

\title{
Non-Perturbative Self-Consistent Model in SU(N) Gauge Field Theory
}

A.V.Koshelkin, a

Kashirskoye sh.,31, Moscow 115409, Russia

\begin{abstract}
Non-perturbative quasi-classical model in a gauge theory with the YangMills (YM) field is developed. The self-consistent solutions of the Dirac equation in the $S U(N)$ gauge field, which is in the eikonal approximation, and the Yang-Mills (YM) equations containing the external fermion current are solved. It shown that the developed model has the self-consistent solutions of the Dirac and Yang-Mills equations at $N \geq 3$. In this way, the solutions take place provided that the fermion and gauge fields exist simultaneously, so that the fermion current completely compensates the current generated by the gauge field due to self-interaction of it.
\end{abstract}

\section{Introduction}

The study of non-Abelian gauge fields plays an important role in the modern field theory[1]. The nonAbelian gauge field are a basis of QCD. The knowledge of solutions of the YM equations enable us to understand specifics of processes in the strong interacting matter generated in collisions of heavy ions of high energies.

The consistent consideration of strong interacting matter (generated, for example, in collisions of high energy ions) demands, generally, solving the Dirac and Yang-Mills equations simultaneously.

In the present paper the quasi-classical model in the $S U(N)$ gauge theory with the Yang Mills field is developed. The self-consistent solution of both the nonhomogeneous Yang-Mills equation and Dirac equations in an external field are derived when the gauge Yang-Mills field is in the eikonal form. It is shown that the self-consistent solutions of such equations take place when $N \geq 3$. They occur provided that the fermion and gauge fields exist simultaneously, so that the fermion current completely compensates the current generated by the gauge field due to its self-interaction.

\section{The YM equations in the presence of external current}

We consider the $S U(N)$ gauge field $A_{a}^{v}$ generated by a fermion current. It satisfies following equations[2]:

$$
\begin{aligned}
& \partial_{\mu} F_{a}^{v \mu}(x)-g \cdot f_{a b}^{c} A_{\mu}^{b}(x) F_{c}^{v \mu}(x)=-g J_{a}{ }^{v}(x) \\
& F_{a}^{v \mu}(x)=\partial^{v} A_{a}^{\mu}(x)-\partial^{\mu} A_{a}^{v}(x)-g \cdot f_{a}^{b c} A_{b}^{v}(x) A_{c}^{\mu}(x), \quad J_{a}^{v}(x)=\bar{\Psi}(x) \gamma^{v} T_{a} \Psi(x),
\end{aligned}
$$

where the fermion fields $\Psi(x)$ is governed by the Dirac equation:

$$
\left\{i \gamma^{\mu}\left(\partial_{\mu}+i g \cdot A_{\mu}^{a}(x) T_{a}\right)-m\right\} \Psi(x)=0 .
$$

\footnotetext{
a e-mail: A Kosh@internets.ru
} 
Here, $m$ is a fermion mass, $g$ is the coupling constant; $\gamma^{v}$ are the Dirac matrixes[2], $x \equiv x^{\mu}=\left(x^{0} ; \mathbf{x}\right)$ is a vector in the Minkowski space-time; $\partial_{\mu}=(\partial / \partial t ; \nabla)$; the Roman letters numerate a basis in the space of the associated representation of the $S U(N)$ group, so that $a, b, c=1 \ldots N^{2}-1$. We use the signature $\operatorname{diag}\left(\mathcal{G}^{\mu v}\right)=(1 ;-1 ;-1 ;-1)$ for the metric tensor $\mathcal{G}^{\mu v}$. Summing over any pair of the repeated indexes is implied. The symbols $T_{a}$ in Eqs.(1)-(2) are the generators of the $S U(N)$ group which satisfy the standard commutative relations and normalization condition[2]. The line over $\Psi(x)$ means the Dirac conjunction.

The main goal is to derive the self-consistent solutions of of Eqs.(1)-(2) which will be localized in the confined region of space. We find the solution when the field $A_{v}^{a}(x)$ is in the form:

$$
A_{a}^{v}(x)=A_{a}^{v}(\varphi(x))
$$

where $\varphi(x)$ is some scalar function in the Minkowski space-time which is such that:

$$
\left(\partial_{\mu} \varphi\right)\left(\partial^{\mu} \varphi\right) \equiv k_{\mu} k^{\mu}=0
$$

The last formula determines the well known eikonal approximation where $\varphi(x)$ can be interpretable as the function governing the wave surface of the field $A_{a}^{v}$.

We take the axial gauge for the field $A_{\mu}^{a}(x)$ :

$$
\partial^{\mu} A_{\mu}^{a}=0 ; \quad k^{\mu} \dot{A}_{\mu}^{a}=0,
$$

where the dot over the letter means differentiation with respect to the introduced variable $\varphi$.

Taking into account of both the dependence of $A_{v}^{a}(x)$ on the variable $x$ via the function $\varphi(x)$ and formulae (4), (5), we derive from Eqs.(1), (2):

$$
\left(\partial_{\mu} \partial^{\mu} \varphi(x)\right) \dot{A}_{a}^{v}+g k^{v} f_{a b}^{c} A_{\mu}^{b}(\varphi) \dot{A}_{c}^{\mu}(\varphi)-g^{2} f_{a b}{ }^{c} f_{c}{ }^{s r}\left\{A_{\mu}^{b}(\varphi) A_{s}^{v}(\varphi) A_{r}^{\mu}(\varphi)\right\}=g J_{a}^{v}(x)
$$

It follows from Eq.(6) that in order to derive the solution of the YM equation it is necessary to calculate the fermion current $J_{a}^{v}(x)$ (see Eqs.(1)). We assume that the field $A_{a}^{v}(\varphi)$ can expanded as follows in the local frame:

$$
\begin{aligned}
& A_{a}^{v}(\varphi)=\mathcal{B}_{a} \partial^{v} \varphi(x)+A\left(e_{(1)}^{v}(\varphi) \cos \left(\varphi(x)+\varphi_{a}\right)+e_{(2)}^{v}(\varphi) \sin \left(\varphi(x)+\varphi_{a}\right)\right) \\
& e_{(1)}^{v} k_{v}=e_{(2)}^{v} k_{v}=0 ; \quad \dot{e}_{(1)}^{v}=e_{(2)}^{v} ; \quad \dot{e}_{(2)}^{v}=-e_{(1)}^{v} ; \quad k^{v} \equiv \partial^{v} \varphi(x),
\end{aligned}
$$

where $e_{(1),(2)}^{v}(\varphi)$ are the space-like 4-vectors on the wave surface $\varphi(x)$ which are independent on the group variable $a$; the symbols $A, \mathcal{B}_{a}$ and $\varphi_{a}$ are the constants in the Minkowski space-time. They are determined via the initial condition of the considered problem. It is obvious that the function $\varphi(x)$ can be taken so that the field $A_{v}^{a}(x)$ will be localized in the confined region of space.

\section{Solution of Dirac equation}

The solution of the Dirac equation (2) when the external field is given by the formula (7) has the following form[3]:

$$
\begin{aligned}
& \Psi_{\sigma, \alpha}(x, p)=\cos \theta \cdot \exp \left(-i g^{2} \frac{\left(N^{2}-1\right) A^{2}}{2 N(p k)} \varphi-i p x\right)\left\{\left(1-i g T_{a} \frac{\tan \theta}{\theta(p k)} \int_{0}^{\varphi} d \varphi^{\prime}\left(A_{\mu}^{a} p^{\mu}\right)\right)+\frac{g\left(\gamma^{\mu} k_{\mu}\right)\left(\gamma^{\mu} A_{\mu}^{a}\right)}{2(p k)}\right. \\
& \left.\left[\frac{\tan \theta}{\theta} T_{a}+\frac{g}{(p k)} \frac{1}{2 N} \int_{0}^{\varphi} d \varphi^{\prime}\left(A_{\mu}^{a} p^{\mu}\right)\left(-i \frac{\tan \theta}{\theta}+\frac{g}{(p k)} \frac{\theta-\tan \theta}{\theta^{3}} T_{b} \int_{0}^{\varphi} d \varphi^{\prime}\left(A_{\mu}^{b} p^{\mu}\right)\right)\right]\right\} u_{\sigma}(p) \cdot v_{\alpha},
\end{aligned}
$$

where $u_{\sigma}(p)$ and $v_{\alpha}$ are some spinors which are the elements of spaces of the corresponding representations. 
Following the structure of the last formula it is naturally to determine the spinor $u_{\sigma}(p)$ as the standard Dirac spinors which satisfy the free Dirac equation and are normalized as follows:

$$
\bar{u}_{\sigma}(p) u_{\lambda}\left(p^{\prime}\right)= \pm 2 m \delta_{\sigma \lambda} \delta_{p p^{\prime}} ; \quad p^{2}=m^{2},
$$

where the plus and minus signs correspond to the Dirac scalar production of the spinors $u_{\sigma}(p)$ and $u_{\sigma}(-p)$, respectively.

As for the spinors $v_{\alpha}$ we determine them by the relations:

$$
v_{\alpha}^{\dagger} v_{\beta}=\delta_{\alpha \beta} ; \operatorname{Tr}\left(T_{a}\right)=0 ; \operatorname{Tr}\left(T_{a} T_{b}\right)=\frac{\delta_{a b}}{2} .
$$

The function (8) can be normalized by the $\delta$-function as follows:

$$
\int d^{3} x \Psi_{\sigma, \alpha}^{*}\left(x, p^{\prime}\right) \Psi_{\sigma, \alpha}(x, p)=(2 \pi)^{3} \delta^{3}\left(\mathbf{p}-\mathbf{p}^{\prime}\right) .
$$

Then, the general solution of the Dirac equation (2) in the external field (7) is:

$$
\Psi(x)=\sum_{\sigma, \alpha} \int \frac{d^{3} p}{\sqrt{2 p^{0}}(2 \pi)^{3}}\left\{\hat{a}_{\sigma, \alpha}(\mathbf{p}) \Psi_{\sigma, \alpha}(x, p)+\hat{b}_{\sigma, \alpha}^{\dagger}(\mathbf{p}) \Psi_{-\sigma, \alpha}(x,-p)\right\},
$$

where the symbols $\hat{a}_{\sigma, \alpha}^{\dagger}(\mathbf{p}) ; \hat{b}_{\sigma, \alpha}^{\dagger}(\mathbf{p})$ and $\hat{a}_{\sigma, \alpha}(\mathbf{p}) ; \hat{b}_{\sigma, \alpha}(\mathbf{p})$ are the operators of creation and cancellation of a fermion $\left(\hat{a}_{\sigma, \alpha}(\mathbf{p}) ; \hat{a}_{\sigma, \alpha}^{\dagger}(\mathbf{p})\right)$ and anti-fermion $\left(\hat{b}_{\sigma, \alpha}(\mathbf{p}) ; \hat{b}_{\sigma, \alpha}^{\dagger}(\mathbf{p})\right)$, respectively, which are governed by the standard commutative relations for fermion operators[2].

\section{Self-consistent consistent solutions of Dirac and Yang-Mills equations}

Substituting Eqs.(7), (12) into the formula (6) we derive:

$$
\begin{aligned}
& 2 f_{a b}{ }^{c} \sin \left(\varphi_{b}-\varphi_{c}\right)=f_{a b}{ }^{c}\left\{f_{c}{ }^{s r} \cos \left(\varphi_{b}-\varphi_{r}\right)+\left\{\cos \left(\varphi_{b}-\varphi_{r}\right) \cos \left(\varphi_{s}-\varphi_{a}\right)\right\} \frac{f_{c}{ }^{b s}}{N}\right\} \mathcal{B}_{s} ; \\
& A^{2} \cdot C=-\left(N^{2}-1\right) \sum_{\sigma \alpha} \int \frac{d^{3} p}{p^{(0)}(2 \pi)^{3}}\left\langle\hat{a}_{\sigma, \alpha}^{\dagger}(\mathbf{p}) \hat{a}_{\sigma, \alpha}(\mathbf{p})+\hat{b}_{\sigma, \alpha}(\mathbf{p}) \hat{b}_{\sigma, \alpha}^{\dagger}(\mathbf{p})\right\rangle, \\
& C=f_{a b}{ }^{c} f_{c}{ }^{s r}\left\{\cos \left(\varphi_{b}-\varphi_{r}\right) \cos \left(\varphi_{s}-\varphi_{a}\right)\right\}<0 .
\end{aligned}
$$

The equations (13) are closed with respect to the unknown quantities $A$ and $\mathcal{B}_{a}$. Having been solved they determine both the fermion and gauge field by means of Eqs.(7), (8), (12), (13) so that the wave surface $\varphi(x)$ is governed by relations (4), (5).

In the case of the $N=2$ the convolution $C$ in Eq.(13)is always positive since the structure constants $f_{a b}{ }^{c}$ are the completely antisymmetrical tensor of the third rang, $\varepsilon_{a b}{ }^{c}$, [2]. This means that in the framework of the developed model there is no self-consistent solution of the Dirac and Yang-Mills equations for the $S U(2)$ gauge symmetry.

As a result, we have the following. The problem governed by Eqs.(1)-(2) has the unique solution when $N \geq 3$. The solutions are determined by (7), (8), (12), (13) and correspond to the eikonal consideration when the wave surface of the fields are determined by the equations:

$$
\left(\partial_{\mu} \varphi(x)\right) \cdot\left(\partial^{\mu} \varphi(x)\right)=0 ;\left(\partial_{\mu} \partial^{\mu}\right) \varphi(x)=0 .
$$

\section{References}

1. P.H.Frampton, Gauge Field Theories, Second Edition, Wiley, 2000.

2. A.I.Akhiezer, S.V.Peletminsky. The field and Fundanmetal Interactions. Kiev, Naukova Dumka, 1986.

3. A.V.Koshelkin. Phys. Lett. B 683 (2010) 205. 1974. 